\title{
Fine Mapping of Qph9, A Major Quantitative Trait Locus, Responsible for Plant Height In Foxtail Millet [Setaria Italica (L.) P. Beauv.]
}

\section{Xiaofen Du ( $\nabla$ dxf6285210@126.com )}

Shanxi Agricultural University https://orcid.org/0000-0003-1185-4477

\section{Zhilan Wang}

Shanxi Agricultural University

\section{Kangni Han}

Shanxi Agricultural University

\section{Shichao Lian}

Shanxi Agricultural University

\section{Yuxin Li}

Shanxi Agricultural University

\section{Linyi Zhang}

Shanxi Agricultural University

\section{Erhu Guo}

Shanxi Agricultural University

Jun Wang

Shanxi Agricultural University

\section{Research Article}

Keywords: Bin-map, Plant height, qPH9,Fine mapping, Foxtail millet

Posted Date: May 21st, 2021

DOI: https://doi.org/10.21203/rs.3.rs-531496/v1

License: (1) (1) This work is licensed under a Creative Commons Attribution 4.0 International License.

Read Full License

Version of Record: A version of this preprint was published at Molecular Breeding on December 1st, 2021. See the published version at https://doi.org/10.1007/s11032-021-01261-w. 


\section{Abstract}

Plant height is vital for crop yield by influencing plant architecture and resistance to lodging. Although lots of quantitative trait loci (QTLs) controlling plant height had been mapped in foxtail millet, their contributions to phenotypic variation were generally small and mapping regions were relatively large, indicating the difficult application in molecular breeding using marker-assisted selection. In the present paper, a total of 23 QTLs involving in 15 traits were identified via a high-density Bin map containing 3024 Bin markers with an average distance of $0.48 \mathrm{cM}$ from an $\mathrm{F}_{2}$ population. Among them, $q P H 9$ with a large phenotypic variation explained (51.6\%) related to plant height, was one of the major QTLs. Furthermore, $q P H 9$ was repeatedly detected in multi-environments under field conditions using two new $\mathrm{F}_{2}$ population from the same $F_{1}$ plant, and was narrowed down to a smaller interval of $281 \mathrm{~kb}$ using 1024 recessive individuals of $F_{2}$ population. Finally, we found that there was an extremely significant correlation between marker MRI1016 and plant height, and speculated that Seita.9G088900 and Seita.9G089700 could be key candidates of $q P H 9$. This study laid an important foundation for the cloning of $q P H 9$ and molecular breeding of dwarf varieties via marker-assisted selection.

\section{Introduction}

Plant height $(\mathrm{PH})$, mainly determined by length of stem internode and number of stem node, is one of important crop yield traits by directly or indirectly affecting heading time, plant structure, lodging resistance, etc. Numerous studies have shown that phytohormones, such as gibberellins (GAs) (Sun 2011), brassinosteroids (BRs) (Clouse et al. 1996), auxin (Multani et al. 2003) and strigolactones (SLs) (Jiang et al. 2013), participate in PH regulation. Mutations in plantohormone biosynthesis or signal transduction may lead to phenotypic variation of $\mathrm{PH}$. For instance, rice mutants of semidwarf1 (OsGA20ox2) and dwarf18 (OsGA30ox2) involving in GAs biosynthesis (Spielmeyer et al. 2002; Itoh et al. 2001), Dwarf1 (OsGID1) and Gibberellin Insensitive Dwarf2 (OsGID2) involving in GAs signal transduction (Ueguchi-Tanaka et al. 2005; Sasaki et al. 2003), exhibited diversely variation in PH. Additionally, PH is also controlled by co-located QTL or pleiotropic genes. In rice, DTH7(Ghd7), encoding a CCT domain protein, played a pleiotropic role for plant height, heading date and grains per panicle (Xue et al. 2008; Gao et al. 2014); Ghd8, encoding a CCAAT box-binding protein which belonged to the HAP3 subfamily, was thought a pleiotropic locus affecting grain yield, heading date, and plant height (Yan et al. 2011).

Application of dwarf lines was thought a main strategy to improve crop yield by increasing the resistance to lodging and maximizing nitrogen-use efficiency (Gooding et al. 2012). During the "Green Revolution", natural variation of the semi-dwarf gene ( $s d-1)$, encoding gibberelin 20-oxidase, was widely used in rice breeding program (Spielmeyer et al. 2002; Sasaki et al. 2002), and dwarfing genes, Rht-1, Rht-2 and Rht-8, were widely applied to wheat (Guo et al. 1997; Worland et al. 1998; Zhang et al. 2006). Foxtail millet, an ancient crop domesticated in China, belongs to a high-density cultivated crop and is prone to lodging, which may lead to a decrease both in yield and quality (Tian et al. 2010). However, there were few 
applications of dwarf lines in production of foxtail millet for lack of efficient genes or QTLs validated in breeding program.

To date, some QTLs responsible for PH had been identified in foxtail millet, such as $q P H 1.1$ (Wang et al. 2017), qph1, qph4, qph5, qph6, qph7 and qph9 (Zhang et al. 2017), qPH1-1, qPH1-2, qPH5-2, qPH5-3, $q P H 8$ and $q P H 9$ (Wang et al. 2019). Nevertheless, their contributions to phenotypic variation were generally minor, and their mapping regions were relatively large, indicating the difficult application in molecular breeding using MAS. Moreover, three candidates including SiD1, SiD2 and Seita.1G242300 were cloned and characterized (Zhao et al. 2019; Xue et al. 2016; He et al. 2021), however to our knowledge there were not any application reports on them in breeding program.

In the present study, QTL mapping was carried out using a high-density Bin map constructed through resequencing strategy in an $F_{2}$ population derived from a cross of two foxtail millet cultivars with low and high plant height, respectively. QTLs for 15 traits were identified, in which $q P H 9$, a major QTL for PH, had been repeatedly validated in two environments. Further, $q P H 9$ was fine mapped onto an interval of $281 \mathrm{~kb}$ through a large recessive $F_{2}$ population. Finally, the InDel marker MRI1016 tightly linked to PH was validated through a analysis of variance (ANOVA), and key candidates of $q P H 9$ were identified via bioinformatics. Our findings provided valuable information for cloning of $q P H 9$ and molecular breeding of dwarf varieties through marker-assisted selection (MAS) in foxtail millet.

\section{Materials And Methods}

Plant materials

To map QTLs for $\mathrm{PH}$, we developed an $\mathrm{F}_{2}$ population derived from a cross between Henggu 12 and Changnong35. Henggu12, the female parent, was characterized by lower biomass, early heading date, lower plant height, multiple tillers and smaller panicle (Li et al. 2015); Changnong35, the male parent, was characterized by higher biomass, late heading date, higher plant height, no tiller and larger panicle (Guo et al. 2008). In 2015, Henggu 12 was crossed with Changnong35 to obtain $F_{1}$, and the true hybrid $F_{1}$ was selected to self-pollinate to generate $F_{2}$ population with 182 plants for preliminary mapping of QTLs for 15 traits in 2017, with 210 plants and 297 plants for verification of $q P H 9$ in two environments, and with 5000 plants, in which 1024 recessive individuals for fine mapping of $q P H 9$, respectively.

The $F_{2}$ individuals and the biparents were grown in row plot with a row length of $2 \mathrm{~m}$, and the density was 30 seeds per row for the $F_{2}$ individuals, and 60 seeds per row for the biparents. In 2017, all materials to map QTLs for 15 traits were sown at Millet Research Institute of Shanxi Agricultural University (MRI), Changzhi $\left(113^{\circ} 08^{\prime} \mathrm{E}, 36^{\circ} 18^{\prime} \mathrm{N}\right)$, China. In 2018 , the materials for $q P H 9$ verification were sown at MRI and the Modern Agricultural Science and Technology Experiment Base, Yuanyang (113 $96^{\prime} \mathrm{E}, 35^{\circ} 05^{\prime}$ $\mathrm{N})$, China, while the materials for fine mapping of $q P H 9$ were sown at MRI. Additionally, the natural population (Supplementary Table S1) for correlation analysis were sown with the same sowing method to biparents described above at MRI in 2018. 
A total of 15 traits were observed in the present study. During maturity, leaf number of the main tiller (LNT), tiller number (TN) and branch number per panicle (BNP) were counted by manual; plant height (PH, $\mathrm{cm})$, main panicle length $(\mathrm{MPL}, \mathrm{cm})$, neck length $(\mathrm{NL}, \mathrm{cm})$, were measured by a ruler; main panicle diameter (MPD, $\mathrm{cm})$ and main stem diameter $(\mathrm{MSD}, \mathrm{cm})$ were measured using a vernier caliper; flag leaf length $(\mathrm{FLL}, \mathrm{cm})$ and flag leaf width $(\mathrm{FLW}, \mathrm{cm})$ were measured using a hand-held laser leaf area meter (YMJ-D, Zhejiang Tuopuyunnong technology Co., Ltd). After harvest, main panicle weight (MPW, g), main grain weight (MGW, g), fresh weight per plant (FWP, g), straw weight per plant (SWP, g) and thousandgrain weight (TGW, g) were measured by electronic balance with an accuracy for $0.01 \mathrm{~g}$. For the natural population, 5 plants for every traits were measured, and the mean value was regarded as the corresponding trait phenotype value. The phenotype data were analyzed using Microsoft Excel 2007 and SPSS Statistics 17.0.

Resequencing and SNP genotyping

Genomic DNA of leaves was isolated using CTAB method (Chen and Ronald 1999). Resequencing was carried out according to the Illumina protocol. Genomic DNA was sheared into 500-base pairs (bp) fragments to construct the library. Paired-end reads (120 bp) were sequenced using the HiSeq 2500 system (Illunima, USA). After filtering the raw reads, the clean reads were aligned to the reference genome of Yugu1 using the Burrows-Wheeler Aligner (BWA) software (Li and Durbin 2009). Genome Analysis ToolKit (GATK) software (McKenna et al. 2010) was used to detect single nucleotide polymorphism (SNP) according to the result of alignment. The Mare Duplicate tool of Picard software (https://sourceforge.net/projects/picard/) was used to remove the duplication. Two SNPs within 5bp or SNPs within $5 \mathrm{bp}$ near InDel were filtered (Reumers et al. 2011). The SNPs identified between the parents were considered as polymorphic for a subsequent Bin calling.

Genetic map construction

For the Bin calling, sliding window method (15 SNPs for each window sliding a site) was used to obtain the genotype. Windows containing more than 13 "aa" or "bb" types were genotyped as "aa" or "bb", respectively. Fifteen adjacent SNP intervals with the same genotype across the entire $F_{2}$ population were combined into a recombination Bin. The Bin markers, within $5 \mathrm{~kb}$ length and with significant segregation distortion ( $\chi^{2}$ test, $P<0.0001$ ), were excluded from the subsequent Bin map construction. Finally, polymorphism Bin markers were obtained to construct the genetic map using the HighMap software (Liu et al. 2014) with default parameters. Marker loci were partitioned primarily into linkage groups (LGs) based on their locations on the reference genome of Yugu1 (https://phytozome.jgi.doe.gov/pz/portal.html\#\%21info?alias=Org_Sitalica_er).

QTL analysis and validation of $q P H 9$ 
QTL analysis was conducted through the Bin map and phenotype of $\mathrm{F}_{2}$ individuals, while validation of $q P H 9$ was carried out basing on genotyping of new developed polymorphic InDel markers on the preliminary mapping region of $q P H 9$ and phenotype of $\mathrm{F}_{2}$ individuals. Interval mapping (IM) analysis with default parameters of Map QTL ${ }^{\circledR} 5$ (Ooijen 2004) was conducted to identify initial candidate QTLs and validate $q P H 9$ for $\mathrm{PH}$. QTLs were called when their LOD values exceeded the genome-wide $95 \%$ confidence threshold. Confidence thresholds were determined through a permutation test (1000 iterations).

InDel marker development and PCR analysis

To fine map $q P H 9$, the flanking sequences, $300 \mathrm{bp}$ upstream and downstream of the insertion-deletion (InDel) loci between biparents across the targeted region, were downloaded and used to develop InDel markers. Primers were designed using primer 3 (http://primer3.ut.ee/).

PCR was conducted in a $10 \mu \mathrm{L}$ reaction volume containing $1 \mu \mathrm{L}$ template DNA (40 ng $\left.\mu \mathrm{L}^{-1}\right), 1.0 \mu \mathrm{L} 10 \times$ reaction buffer, $0.8 \mu \mathrm{L} 10 \mathrm{mmolL}^{-1} \mathrm{dNTPs}, 2.0 \mu \mathrm{L}$ primer $\left(2 \mu \mathrm{m} \mathrm{L}^{-1}\right), 0.1 \mu \mathrm{L}$ rTaq DNA polymerase

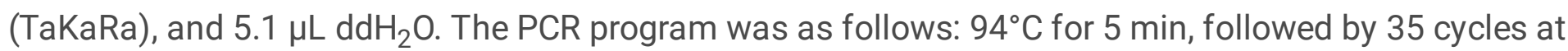
$94^{\circ} \mathrm{C}$ for $25 \mathrm{~s}, 58^{\circ} \mathrm{C}$ for $25 \mathrm{~s}$, and $72^{\circ} \mathrm{C}$ for $20 \mathrm{~s}$, and terminated by a final extension for 10 min at $72^{\circ} \mathrm{C}$. The fragments of PCR were electrophoresed on $8 \%$ non-denatured polyacrylamide gel electrophoresis (PAGE) and visualized by silver staining (Marklund et al. 1995).

Fine mapping and candidate analysis of $q P H 9$

To narrow down the $q P H 9$ interval, 1024 recessive $\mathrm{F}_{2}$ individuals (dwarf plants) were genotyped with polymorphic InDel markers, "a" represented the Henggu12 genotype, "b" represented the Changnong35 genotype, and " $h$ " was the heterozygous genotype. In the process of screening recessive $F_{2}$ individuals, "b" or " $h$ " indicated the recombinants. Genes within the fine-mapped region were annotated according to Yugu1 genome. Gene expression information was obtained from MDSi database (http://foxtailmillet.biocloud.net/page/tools/expressionVisualization).

\section{Results}

Phenotypic and correlation analysis

In this study, a total of 15 traits were evaluated (Supplementary Table S2) among $F_{2}$ individuals (182 plants) and biparents. The phenotypic variations for each trait had a wide range with a large variation coefficient, and the absolute value of the skewness and kurtosis of most traits was $<1$, indicating that these traits had great potentials for genetic improvement. Additionally, these traits exhibited a dramatic distributions in $F_{2}$ population: apart from NL with a normal distribution, $P H, L N T, M P L, M P D, M S D, F L L$, FLW, TGW and BNP had a distinct bimodal distribution, and FWP, SWP, MPW, MGW and TN deviated from a normal distribution (Fig. 1). 
The Pearson correlation coefficient with bilateral detection was performed across 15 traits (Supplementary Table S3). TN with the other traits showed negative correlation; NL with PH, MPL, MPD, MSD, BNP, FLL, FLW, FWP, SWP, MPW, MGW and TGW showed negative correlation; positive correlation was observed among the other traits. Even some traits exhibited extremely significant correlation. For instance, the correlation coefficients between PH and MPL, MSD, LNT, FLL and MSD were 0.960, 0.941, 0.928 and 0.901 , respectively; LNT and MPL, MSD and FLL were 0.937, 0.925 and 0.918, respectively; MPL and FLL were 0.943; MPD and MSD were 0.916, indicating these traits might be affected by a pleiotropic gene or closely linked loci.

Construction of the high density genetic map

Biparents and $182 \mathrm{~F}_{2}$ individuals were sequenced in the present study. Totally, we obtained $8.08 \mathrm{Gbp}$ clean data for Henggu12 with an average coverage 17x, $7.38 \mathrm{Gbp}$ for Changnong35 with an average coverage $16 x$, and 278.38 Gbp for offspring with an average coverage 3.23x. The genetic map was constructed using 3024 Bin markers originated from 651,047 SNPs, and the distance of the map was $1457.40 \mathrm{cM}$ with an average distance of $0.48 \mathrm{cM}$ between adjacent Bin markers (Table 1; Supplementary Fig. S1; Supplementary Table S4). Interestingly, compared with other chromosomes, Chr. VIII shared the largest number of markers, the maximum length of total distance, the minimum length of average distance, and the minimum gap. Additionally, colinearity analysis showed that the genetic map reported here had a good collinearity with the reference genome (Fig. S2).

QTL analysis for 15 traits

MapQTL was carried out to detect QTLs controlling 15 traits. A total of 23 QTLs were identified on Chr. VIII (Table 2). Collectively, only one QTL was identified for PH, LNT, MSD, FLL, FLW, FWP, SWP, MGW, TGW and BNP, with a PVE from 31.2-54.5\%, an additive effect from - 0.22 to -56.92 , and a dominate effect from 0.38 to 116.76, respectively; two QTLs were identified for MPL, MPD, MPW and NL, respectively, in which qMPL9-2 for MPL and qMPD9-2 for MPD were two major effect QTLs and accounted for $47.9 \%$ and $45.4 \%$ of the PVE, respectively; for TN, 5 QTLs (qTN9-1, qTN9-2, qTN9-3, qTN9-4 and qTN9-5) with a PVE from $12.2-20.7 \%$, an additive effect from 0.42 to 0.59 , and a dominate effect from -0.44 to -0.65 were detected, respectively. Notably, $q P H 9$ with a large PVE (51.6\%), a major QTL for PH, was observed and shared the same position with $q M S D 9$ and $q F L L 9$, in charge of MSD and FLL, respectively.

Validation of $q P H 9$

To verify $q P H 9$, QTL analysis was conducted using the $\mathrm{F}_{2}$ plants (210 plants and 297 plants) from the same $F_{1}$ individual in two environments (Changzhi and Yuanyang, China). A total of 12 InDel markers (Supplementary Table S5), showing polymorphisms between two parental lines in the candidate region of $q P H 9$, were used to validate $q P H 9$. The results showed that the distinct bimodal distribution for $\mathrm{PH}$ was repeatedly observed in two environments, indicating that $\mathrm{PH}$ was controlled by a major QTL or gene, and that $q P H 9$ was repeatedly detected and shared the same chromosome position in two environments, although there were some differences in LOD score and PVE, which were 66.69 and $79 \%$ in Changzhi 
(Fig. 2a; Supplementary Table S6), 92.9 and 76.4\% in Yuanyang (Fig. 2b; Supplementary Table S7), respectively. Furthermore, ANOVA analysis revealed that there was an extremely significant correlation between MRI1016 and $\mathrm{PH}$ not only in $\mathrm{F}_{2}$ population in two environments, but also in the natural population (Supplementary Table S8).

Fine mapping and candidate analysis of $q P H 9$

To narrow down the mapping interval of $q P H 9,13$ polymorphism InDel markers (Supplementary Table S5) were developed from its preliminary mapping physical region (Fig. 3a), and 1024 recessive $F_{2}$ individuals (dwarf plants) from the same $F_{1}$ derived from the cross between Henggu12 and Changnong35 were randomly selected and screened for recombinants. The results showed that the number of recombinants for 13 polymorphism InDel markers were 270 (MRI480), 147 (MRI548), 79 (MRI819), 40 (MRI1010), 10 (MRI1014), 6 (MRI1016), 10 (MRI1018), 10 (MRI1019), 57 (MRI1058), 80 (MRI954), 100 (MRI627), 220 (MRI557) and 365 (MRI559), respectively, indicating that $q P H 9$ was located on the interval of $281 \mathrm{~kb}$ between MRI1104 (5,314,541) and MRI1108 (5,595,508) (Fig. 3b).

According to the reference genome of Yugu1, 45 genes were found in this region $(5,314,541-5,595,508)$, of which 36 genes were annotated. Among the annotated, 9 genes, including Seita.9G088400, Seita.9G088500, Seita.9G088900, Seita.9G089000, Seita.9G089700, Seita.9G09100, Seita.9G0901200, Seita.9G091400 and Seita.9G092100, showed higher expression in stem of Xiaomi and JG21 according to the fragments per kilobase million (FPKM), of which four genes, consisting of Seita.9G088400, Seita.9G088500, Seita.9G088900 and Seita.9G089700, were homologous to OsBC1L4, ZmPHYC, OsMADS14 and AtAGL7 involving in $\mathrm{PH}$ in rice, maize and Arabidopsis, respectively (Supplementary Table S9). Combining with the analysis of recombinants of $13 \mathrm{InDel}$ markers, we knew that Seita. 9 G088400 $(5,317,918-5,321,537)$ and Seita. 9 G088500 $(5,322,108-5,326,090)$ were located near the marker MRI1014 $(5,314,541)$ with 10 recombinant plants; Seita. 9 G088900 $(5,365,359-5,373,122)$ and Seita.9G089700 $(54,121,575-5,416,960)$ were located near the marker MRI1016 $(5,490,668)$ with 6 recombinant plants. Thus we speculated that Seita. 9 G089700 and Seita. 9 G088900 could be better candidates for $q P H 9$.

\section{Discussion}

As cost of genome sequencing became decreased, QTL mapping for complex traits, and also for the quality-quantity traits, has become extremely effective by SNP strategy based on next-generation sequencing (NGS) technology. Previously, in Capsella rubella, CrFLC controlling flowering time, as a quality-quantity gene, was identified and cloned in two distinct $F_{2}$ population (Yang et al. 2018), and qCOK2 with a PVE of $31.9 \%$ for color of kernel was detected and fine mapped through an $\mathrm{F}_{2}$ population in maize (Wang et al. 2018). In this study, we preliminarily mapped, repeatedly validated and fine mapped the $q P H 9$ using four different $\mathrm{F}_{2}$ population from the same $\mathrm{F}_{1}$ derived from a cross between Henggu 12 and Changnong35. Inspiringly, $q P H 9$ could be repeatedly detected in multi-environments, and finally it was fine mapped onto a smaller interval of $281 \mathrm{~kb}$, indicating that it was effective to fine map QTLs for 
quality-quantity trait using several different $F_{2}$ mapping population from an $F_{1}$ individual. These results provided a further insight on fine mapping of QTLs associated with quality-quantity trait.

In the present study, we identified 23 QTLs for 15 traits using resequencing strategy. Dramatically, extreme QTL cluster for all QTLs except $q N L 9-1$ and $q N L 9-2$ were observed. This phenomenon was also found and thought to be associated with ecological significance in wild annuals of rice (Onishi 2007). In this study, biparents had distinct features in multi-traits associated with ecological significance including heading date, sensitive to light and temperature, plant height, etc. Hengu12 could be grown across China and the performance could be almost normal due to its shorter heading date and insensitive to light and temperature ( $\mathrm{Li}$ et al. 2015), but Changnong 35 just in north China, indicating that the QTL cluster on Chr. VIII might be related to ecological adaptability in foxtail millet. Also, we found all QTLs for 15 traits were mapped onto Chr. VIII and none was present on the other chromosomes. This phenomenon might be resulted from those causes as follows: (1) Quality-quantity traits: almost all traits exhibited a distinct bimodal distribution (Fig. 1), indicating that these traits could be of quality-quantity traits in this population. Due to the nature of quality-quantity traits, major QTLs were easily detected and the minor QTLs were difficultly detected in $\mathrm{F}_{2}$ mapping population; (2) Extremely significant correlation: this was found almost among all traits (Supplementary Table S3), indicating major QTLs responsible for those traits might be tightly linked together each other; (3) Ecological adaptability illustrated above. To this end, it was necessary to develop NIL population to further map minor QTLs.

QTL co-location, meaning that QTLs responsible for correlative traits are often co-located in a specific interval, is a widespread phenomenon in plant genomes. Co-located QTL was usually used to assess the selective genetic effects, or to understand pathways of genetic activity for breeders and geneticists (Hill and Zhang 2012). In foxtail millet, previous studies showed that this phenomenon also existed (Zhang et al. 2017; Wang et al. 2019; Liu et al. 2020; Mauro-Herrera and Doust 2016; Fang et al. 2016). Generally, co-located QTLs were caused by pleiotropy, such as Q gene responsible for free-threshing, spike rachis fragility, plant height, spike shape and heading date (Simons et al. 2006), and Rht-B1 gene responsible for plant height, grain size and weight (Guan et al. 2020) in wheat, or close linkage of different genes affecting multiple traits such as Dw2 and Ma1 in sorghum in charge of plant height and flowering time, respectively (Klein et al. 2008). In the present study, QTLs controlling PH, MSD and FLL with large effects were also co-located onto the same region on Chr. VIII, which was consistent with significant positive phenotypic correlation between these traits, indicating that $q P H 9$ might be a pleiotropic QTL.

In this study, $q P H 9$ was fine mapped onto the interval of 5,314,541-5,595,508 between InDel marker MRI1104 and MRI1108. Previous studies showed that 9 QTLs for PH on Chr. VIII, consisting of H9a: 3,452,395-9,964,754 (Mauro-Herrera and Doust 2016), qph9: 1,559,957-1,859,997 (Zhang et al. 2017), qPH9 8 8,207,836-8,386,583 (Wang et al. 2019), qPH9.1: 31,657,629-3,379,493, qPH9.2: 35,624,88739,708,798, qPH9.3: 41,536,123 - 42,595,374, qPH9.4: 42,767,054 - 43,133,505, qPH9.5: 43,430,778 43,849,397 and $q P H 9.6: 46,893,323-47,598,99$ (He et al. 2021), had been detected in foxtail millet. Distinctly, except for H9a, physical regions of the other QTLs were different from ours $(5,314,541$ $5,595,508)$. Notably, $H 9 a$, had been repeatedly detected not only at different growth stages but also in 
multi-environments (Mauro-Herrera and Doust 2016), in spite of the physical region was distinctly larger than $q P H 9$ in this study. Interestingly, $q P H 9$ was also repeatedly detected in multi-environments, and had the similar PVE from $76.4-79 \%$ in different environments, indicating that $q P H 9$ was an stable and high hereditary locus. Indubitably, there have been one or several stably major QTLs on Chr. VIII responsible for $\mathrm{PH}$, and $q P H 9$ in the present study was the finest one mapped so far.

To analyse the candidate genes for $q P H 9$, we searched for the putative functions of genes across the physical region of $q P H 9$ by Phytozome database. We found that there were 36 annotated genes within the physical region of $q P H 9$, of which 8 genes, including Seita.9G088400, Seita.9G088500, Seita.9G088900, Seita.9G089000, Seita.9G090100, Seita.9G091200, Seita.9G091400 and Seita.9G092100 exhibited higher expression level in stem either in Xiaomi or in JG21, and one, Seita.9G089700, exhibited higher expression both in Xiaomi and in JG21 (Supplementary Table S9). Bioinformatics analysis showed that Seita.9G088900 was homologous to OSMADS14 and AtAGL7 regulating the development of inflorescence meristem and flowering in rice and Arabidopsis, respectively (Jeon et al. 2000; Han et al. 2014), and Seita.9G089700 was homologous to ZmPHYC involving in flowering time and plant height in maize ( $\mathrm{Li}$ et al. 2020). Moreover, analysis of recombinant plants near the markers MRI1014 $(5,314,541)$ and MRI1016 $(5,490,668)$ showed that MRI1016 has the smaller number of recombinant plants. We therefore suggested Seita.9G088900 and Seita.9G089700 might be the key candidates.

\section{Conclusion}

In the present study, we identified a stable major locus ( $q P H 9$ ) controlling plant height in multienvironments, and further fine mapped it onto an interval of $281 \mathrm{~kb}$. Moreover, we developed an InDel marker MRI1016 tightly linked to PH and found that Seita.9G088900 and Seita.9G089700 might be key candidates of $q P H 9$. These results were conducive to cloning of $q P H 9$ and to molecular breeding of dwarf varieties via MAS in foxtail millet.

\section{Abbreviations}

PH plant height

MPL main panicle length

MPD main panicle diameter

MSD main stem diameter

MPW main panicle weight

MGW main grain weight

FWP fresh weight per plant 
SWP straw weight per plant

TGW thousand-grain weight

FLL flag leaf length

FLW flag leaf width

LNT leaf number of the tallest tiller

TN tiller number

$\mathrm{NL}$ neck length

BNP branch number per panicle

QTLs quantitative trait loci

MAS marker-assisted selection

PVE phenotypic variation explained

NGS next-generation sequencing

RAD-seq restriction site-associated DNA sequencing

FPKM fragments per kilobase million

SNP single nucleotide polymorphism

InDel insertion-deletion

\section{Declarations}

\section{Funding}

This research was supported by National Key R\&D Program of China (2018YFD1000700), National Youth Science Foundation of China (32001609), Minor Crop Molecular Breeding Platform Special Project of Shanxi Academy of Agricultural Sciences (YGC2019FZ3), Research Project Supported by Shanxi Scholarship Council of China (HGKY2019101) and Agricultural Science and Technology Innovation Research Project of Shanxi Academy of Agricultural Sciences (YCX2020YQ35).

\section{Conflicts of interest}

The authors declared no conflict of interest.

\section{Availability of data and material}


Raw sequence data of biparents and $182 \mathrm{~F}_{2}$ individuals are not publicly available due to the research of another important trait, but are available from the corresponding author on reasonable request. The other data generated or analysed in this study are included in this manuscript and its supplementary information files.

\section{Code availability}

Not applicable

\section{Ethics approval}

Not applicable

\section{Consent to participate}

Not applicable

Consent for publication

Not applicable

\section{Authors' contributions}

JW conceived and supervised the complete study; XFD and ZLW performed the experiments and carried out the bioinformatics work; EHG, SCL, KNH, YXL and LYZ were responsible for field trial; XFD and JW wrote and revised the manuscript. All authors have read and approved the final manuscript.

\section{References}

Chen DH, Ronald PC (1999) A rapid DNA minipreparation method suitable for AFLP and other PCR applications. Plant Mol Biol Rep 17:53-57

Clouse SD, Langford M, Mcmorris TC (1996) A brassinosteroid-insensitive mutant in Arabidopsis thaliana exhibits multiple defects in growth and development. Plant Physiol 111:671-678

Fang XM, Dong KJ, Wang XQ et al (2016) A high density genetic map and QTL for agronomic and yield traits in Foxtail millet [Setaria italica (L.) P. Beauv]. BMC Genom 17:336. https://doi:10.1186/s12864-016$2628-z$

Gao H, Jin MN, Zheng XM et al (2014) Days to heading 7, a major quantitative locus determining photoperiod sensitivity and regional adaptation in rice. Proc Natl Acad Sci U S A. 111: 16337-16342. https://doi:10.1073/pnas.1418204111

Gooding MJ, Addisu M, Uppal RK, Snape JW, Jones HE (2012) Effect of wheat dwarfing genes on nitrogen-use efficiency. The Journal of Agricultural Science 150:3-22. 
https://doi:10.1017/s0021859611000414

Guan PF, Shen XY, Mu Q et al (2020) Dissection and validation of a QTL cluster linked to Rht-B1 locus controlling grain weight in common wheat (Triticum aestivum L.) using near-isogenic lines. Theor Applied Genet 133: 2693-2653. https://doi: 10.1007/s00122-020-03622-z

Guo BH, Song CH, Jia JZ (1997) Identification and distribution of and dwarf genes in Chinese elite dwarf wheat varieties. Scientia Agricultura Sinica 30:56-60

Guo EH, Fan HP, Wang XQ, Cheng LP, Wang J, Guo HL, Wang QL (2008) Breeding of new variety Changnong35 in foxtail millet. Chin Agric Sci Bull 24:188-191

Han YY, Zhang C, Yang HB, Jiao YL (2014) Cytokinin pathway mediates APETALA1 function in the establishment of determinate floral meristems in Arabidopsis. Proc Natl Acad Sci U S A 111:6840-6845. doi 10.1073/pnas.1318532111

He Q, Zhi H, Tang S et al (2021) QTL mapping for foxtail millet plant height in multi-environment using an ultra-high density bin map. Theor Appl Genet 134:557-572. https://doi:10.1007/s00122-020-03714-w

Hill WG, Zhang XS (2012) On the pleiotropic structure of the genotype-phenotype map and the evolvability of complex organisms. Genetics 190:1131-1137. https://doi:10.1534/genetics.111.135681

Itoh H, Ueguchi-Tanaka M, Sentoku N, Kitano H, Matsuoka M, Kobayashi M (2001) Cloning and functional analysis of two gibberellin 3-hydroxylase genes that are differently expressed during the growth of rice. Proc Natl Acad Sci U S A 98:8909-8914. https://doi:10.1073/pnas.141239398

Jeon JS, Lee S, Jung KH, Yang WS, Yi GH, Oh BG, An G (2000) Production of transgenic rice plants showing reduced heading date and plant height by ectopic expression of rice MADS-box genes. Mol Breeding 6:581-592

Jiang L, Liu X, Xiong GS et al (2013) DWARF 53 acts as a repressor of strigolactone signalling in rice. Nature 2013: 504, 401-405. https://doi:10.1038/nature12870

Klein RR, Mullet JE, Jordan DR, Miller FR, Rooney WL, Menz MA, Franks CD, Klein PE (2008) The effect of tropical sorghum conversion and inbred development on genome diversity as revealed by high-resolution genotyping. Crop Sci 48:S12-S26. https://doi:10.2135/cropsci2007.06.0319tpg

Li H, Durbin R (2009) Fast and accurate short read alignment with Burrows-Wheeler transform. Bioinformatics 25:1754-1760. doi:10.1093/bioinformatics/btp324

Li QQ, Wu GX, Zhao YP, Wang BB, Zhao BB, Kong DX, Wei HB, Chen CX, Wang HY (2020) CRISPR/Cas9mediated knockout and overexpression studies reveal a role of maize phytochrome $\mathrm{C}$ in regulating flowering time and plant height. Plant Biotechnol J 18:2520-2532. https://doi:10.1111/pbi.13429 
Li MZ, Hao HB, Liu GB, Xie N, Cui HY (2015) Breeding of new summer foxtail millet variety Henggu No.12 with dwarf and eariliest-maturing. Journal of Hebei Agricultural Sciences 19:3-5.

https://doi:10.16318/j.cnki.hbnykx.2015.06.002

Liu D, Ma C, Hong W, Huang L, Liu M, Liu H, Zeng H, Deng D, Xin H, Song J, Xu C, Hou X, Wang X, Zheng H (2014) Construction and analysis of high-density linkage map using high-throughput sequencing data. Plos One 9:e98855. https://doi:10.1371/journal.pone.0098855

Liu TP, He JH, Dong KJ et al (2020) QTL mapping of yield component traits on bin map generated from resequencing a RIL population of foxtail millet (Setaria italica). BMC Genomics 21:141. https://doi:10.1186/s12864-020-6553-9

Marklund S, Chaudhary R, Marklund L, Sandberg K, Andersson L (1995) Extensive mtDNA diversity in horses revealed by PCR-SSCP analysis. Animal genetics 26:193-196

Mauro-Herrera M, Doust AN (2016) Development and genetic control of plant architecture and biomass in the panicoid grass, Setaria. Plos One 11:e0151346. https://doi:10.1371/journal.pone.0151346

McKenna A, Hanna M, Banks E et al (2010) The Genome Analysis Toolkit: a MapReduce framework for analyzing next-generation DNA sequencing data. Genome Res 20:1297-1303.

https://doi:10.1101/gr.107524.110

Multani DS, Briggs SP, Chamberlin MA, Blakeslee JJ, Murphy AS, Johal GS (2003) Loss of an MDR transporter in compact stalks of Maize br2 and Sorghum $d w 3$ mutants. Science 302:81-84. doi $10.1126 /$ science. 1086072

Onishi K, Horiuchi Y, Ishigoh-Oka N, Takagi K, Ichikawa N, Maruoka M, Yoshio S (2007) A QTL culster for plant architecture and its ecological significance in Asian wild rice. Breed Sci 57:7-16

Ooijen JW. MapQTL5.0, software for the mapping of quantitative trait loci in experimental populations. Kyazma BV: Wageningen, The Netherlands, 2004

Reumers J, De Rijk P, Zhao H et al (2011) Optimized filtering reduces the error rate in detecting genomic variants by short-read sequencing. Nat Biotechnol 30:61-68. https://doi:10.1038/nbt.2053

Sasaki A, Ashikari M, Ueguchi-Tanaka M, Itoh H, Nishimura A, Swapan D, Ishiyama K, Saito T, Kobayashi M, Khush GS, Kitano H, Matsuoka M (2002) Green revolution: a mutant gibberellin-synthesis gene in rice. Nature 416:701-702. doi 10.1038/416701a

Sasaki A, Itoh H, Gomi K, Ueguchi-Tanaka M, Ishiyama K, Dh KMJ, Kitano H, Ashikari M, Matsuoka M (2003) Accumulation of phosphorylated repressor for gibberellin signaling in an F-box mutant. Science 299:1896-1898. doi 10.1126/science.1081077 
Simons KJ, Fellers JP, Trick HN, Zhang Z, Tai Y-S, Gill BS, Faris JD (2006) Molecular characterization of the major wheat domestication gene Q. Genetics 172:547-555. https://doi:10.1534/genetics.105.044727

Spielmeyer W, Ellis MH, Chandler PM (2002) Semidwarf (sd-1), "green revolution" rice, contains a defective gibberellin 20-oxidase gene. Proc Natl Acad Sci U S A. 99: 9043-9043. doi: 10.1073/pnas.132266399

Sun TP (2011) The molecular mechanism and evolution of the GA-GID1-DELLA signaling module in plants. Curr Biol 21: R338-R345. doi: 10.1016/j.cub.2011.02.036

Tian BH, Wang JG, Zhang LX, Li YJ, Wang SY, Li HJ (2010) Assessment of resistance to lodging of landrace and improved cultivars in foxtail millet. Euphytica 172:295-302. https://doi:10.1007/s10681009-9999-z

Ueguchi-Tanaka M, Ashikari M, Nakajima M, Itoh H, Katoh E, Kobayashi M, Chow TY, Hsing Y, Kitano H, Yamaguchi I (2005) GIBBERELLIN INSENSITIVE DWARF1 encodes a soluble receptor for gibberellin. Nature 437:693-698. doi:10.1038/nature04028

Wang J, Wang ZL, Du XF et al (2017) A high-density genetic map and QTL analysis of agronomic traits in foxtail millet [Setaria italica (L.) P. Beauv.] using RAD-sEq. Plos One 12:e0179717.

https://doi:10.1371/journal.pone.0179717

Wang J, Zhang X, Lin ZW (2018) QTL mapping in a maize $F_{2}$ population using Genotyping-bySequencing and a modified fine-mapping strategy. Plant Sci 276:171-180. https://doi:10.1016/j.plantsci.2018.08.019

Wang ZL, Wang J, Peng JX et al (2019) QTL mapping for 11 agronomic traits based on a genome-wide Bin-map in a large $F_{2}$ population of foxtail millet (Setaria italica (L.) P. Beauv). Mol Breeding 39:18. https://doi:10.1007/s11032-019-0930-6

Worland AJ, Korzun V, Röder MS, Ganal MW, Law CN (1998) Genetic analysis of the dwarfing gene Rht8 in wheat. Part II. The distribution and adaptive significance of allelic variants at the Rht8 locus of wheat as revealed by microsatellite screening. Theor Appl Genet 96:1110-1120

Xue CX, Zhi H, Fang XJ, Liu XT, Tang S, Chai Y, Zhao BH, Jia GQ, Diao XM (2016) Characterization and fine mapping of SiDWARF2 (D2) in foxtail millet. Crop Sci 56:95-103.

https://doi:10.2135/cropsci2015.05.0331

Xue WY, Xing YZ, Weng XY, Zhao Y, Tang WJ, Wang L, Zhou HJ, Yu SB, Xu CG, Li XH, Zhang QF (2008) Natural variation in Ghd7 is an important regulator of heading date and yield potential in rice. Nat Genet 40:761-767. https://doi:10.1038/ng.143

Yan WH, Wang P, Chen HX, Zhou HJ, Li QP, Wang CR, Ding ZH, Zhang YS, Yu SB, Xing YZ, Zhang QF (2011) A Major QTL, Ghd8, plays pleiotropic roles in regulating grain productivity, plant height, and 
heading date in rice. Mol Plant 4:319-330. doi:10.1093/mp/ssq070

Yang L, Wang HN, Hou XH, Zou YP, Han TS, Niu XM, Zhang J, Zhao Z, Todesco M, Balasubramanian S, Guo YL (2018) Parallel evolution of common allelic variants confers flowering diversity in Capsella rubella. Plant Cell 30:1322-1336. https://doi:10.1105/tpc.18.00124

Zhang K, Fan GY, Zhang XX et al (2017) Identification of QTLs for 14 agronomically important traits in Setaria italica based on SNPs generated from high-throughput sequencing. G3 7: 1587-1594. https://doi:10.1534/g3.117.041517/-/DC1

Zhang X, Yang S, Zhou Y, He Z, Xia X (2006) Distribution of the Rht-B1b, Rht-D1b and Rht8 reduced height genes in autumn-sown Chinese wheats detected by molecular markers. Euphytica 152: 109-116. https://doi: 10.1007/s10681-006-9184-6

Zhao MC, Zhi H, Zhang X, Jia GQ, Diao XM (2019) Retrotransposon-mediated DELLA transcriptional reprograming underlies semi-dominant dwarfism in foxtail millet. The Crop Journal 7:458-468. https://doi:10.1016/j.cj.2018.12

\section{Tables}

Due to technical limitations, table 1 and 2 is only available as a download in the Supplemental Files section.

\section{Figures}



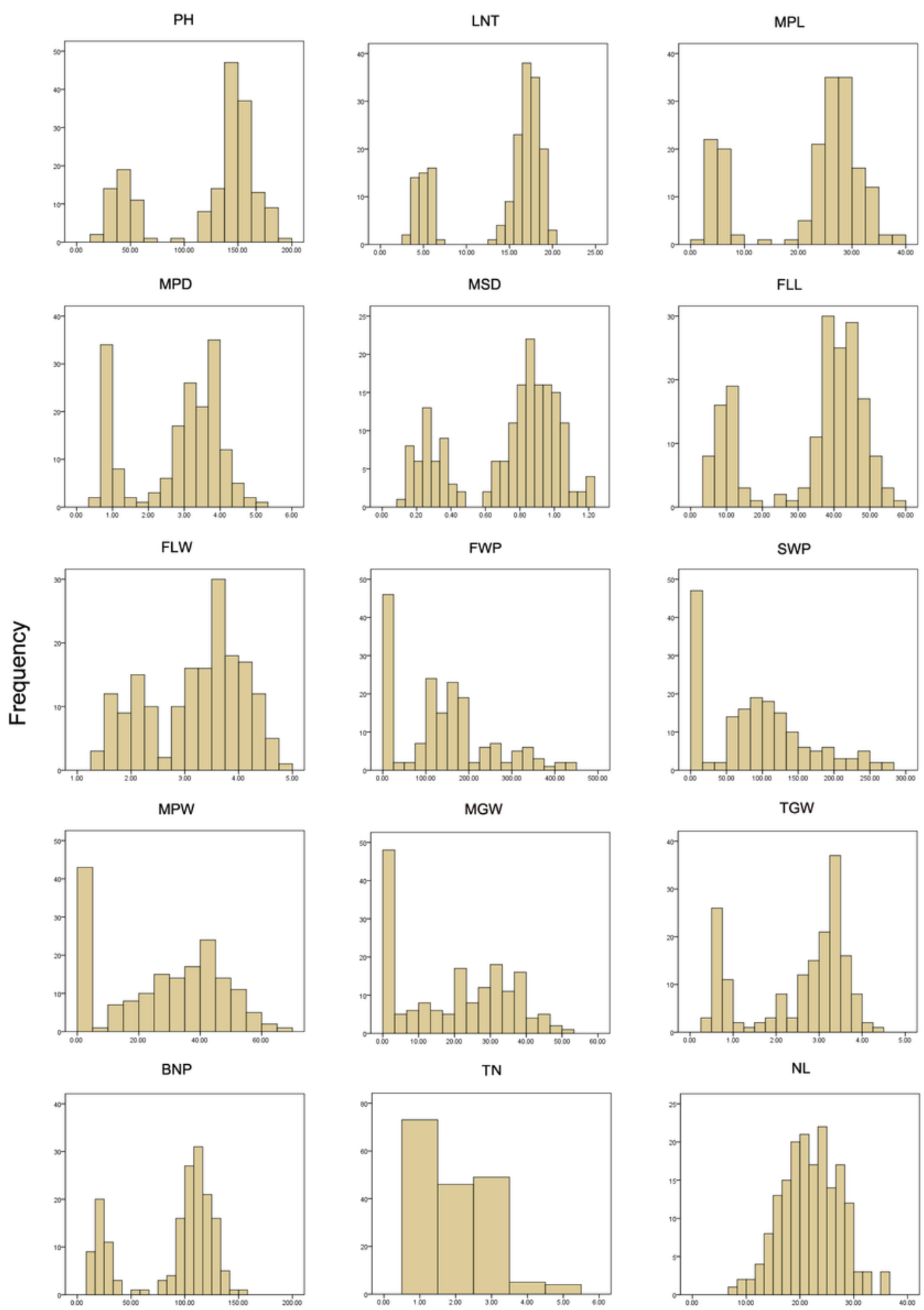

NL

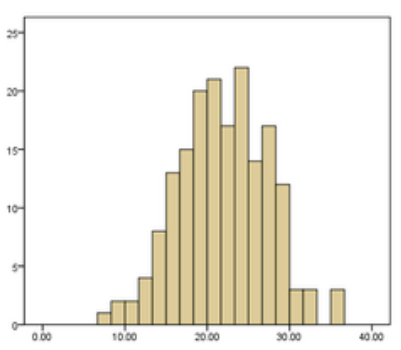

Figure 1

Distribution of 15 traits in the F2 population derived from a cross between Henggu12 and Changnong35 

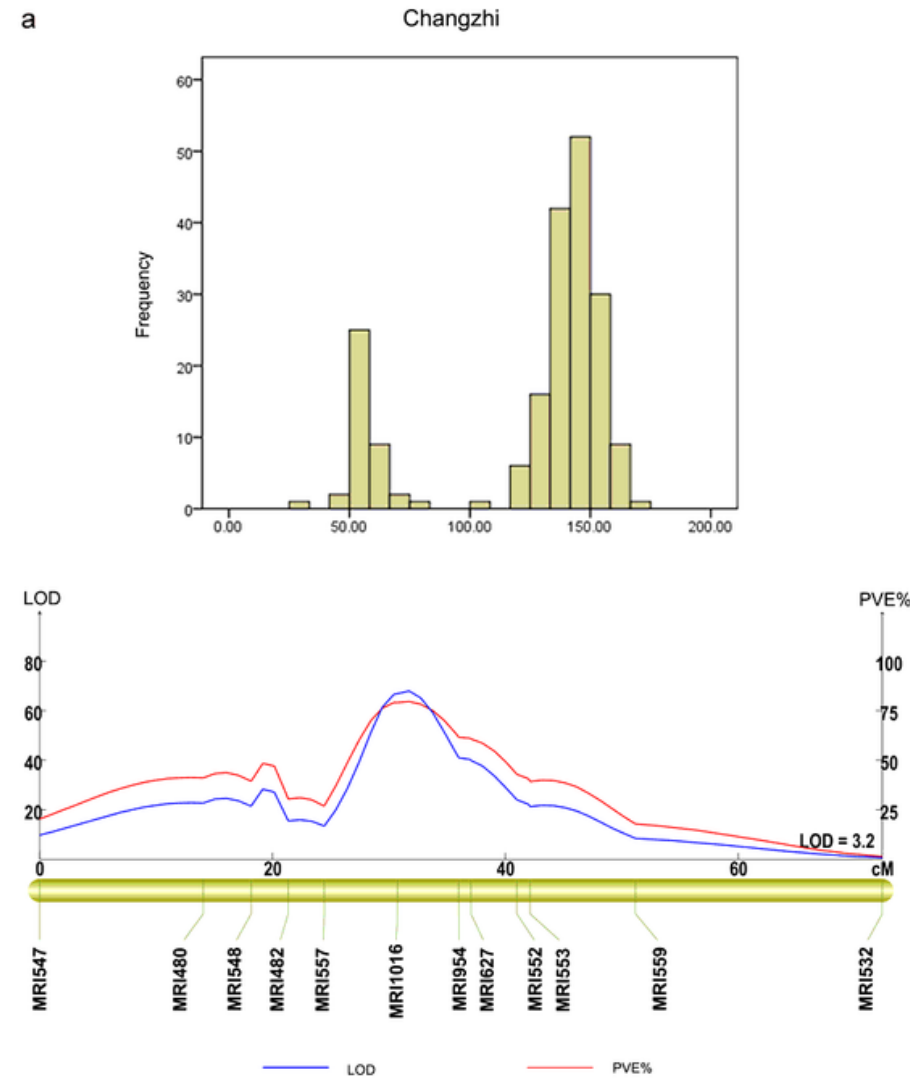
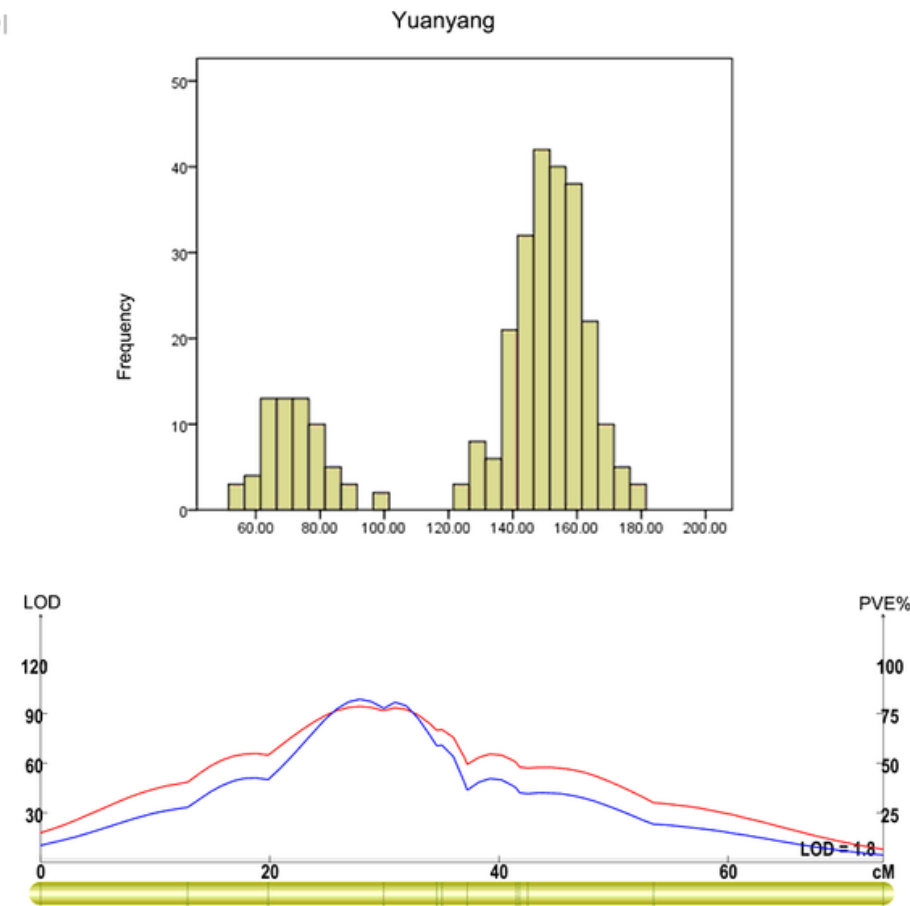

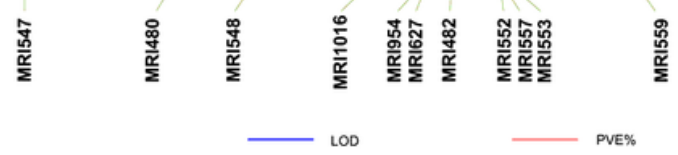

Figure 2

Validation of qPH9 in two environments. a The distribution of $210 \mathrm{~F} 2$ individuals and genetic linkage analysis with InDel markers in Changzhi, scale of Y-axis showed LOD value and scale of X-axis showed centimorgan (cM). b The distribution of 297 F2 individuals and genetic linkage analysis with InDel markers in Yuanyang, scale of Y-axis showed LOD value and scale of X-axis showed centimorgan (cM) 
a

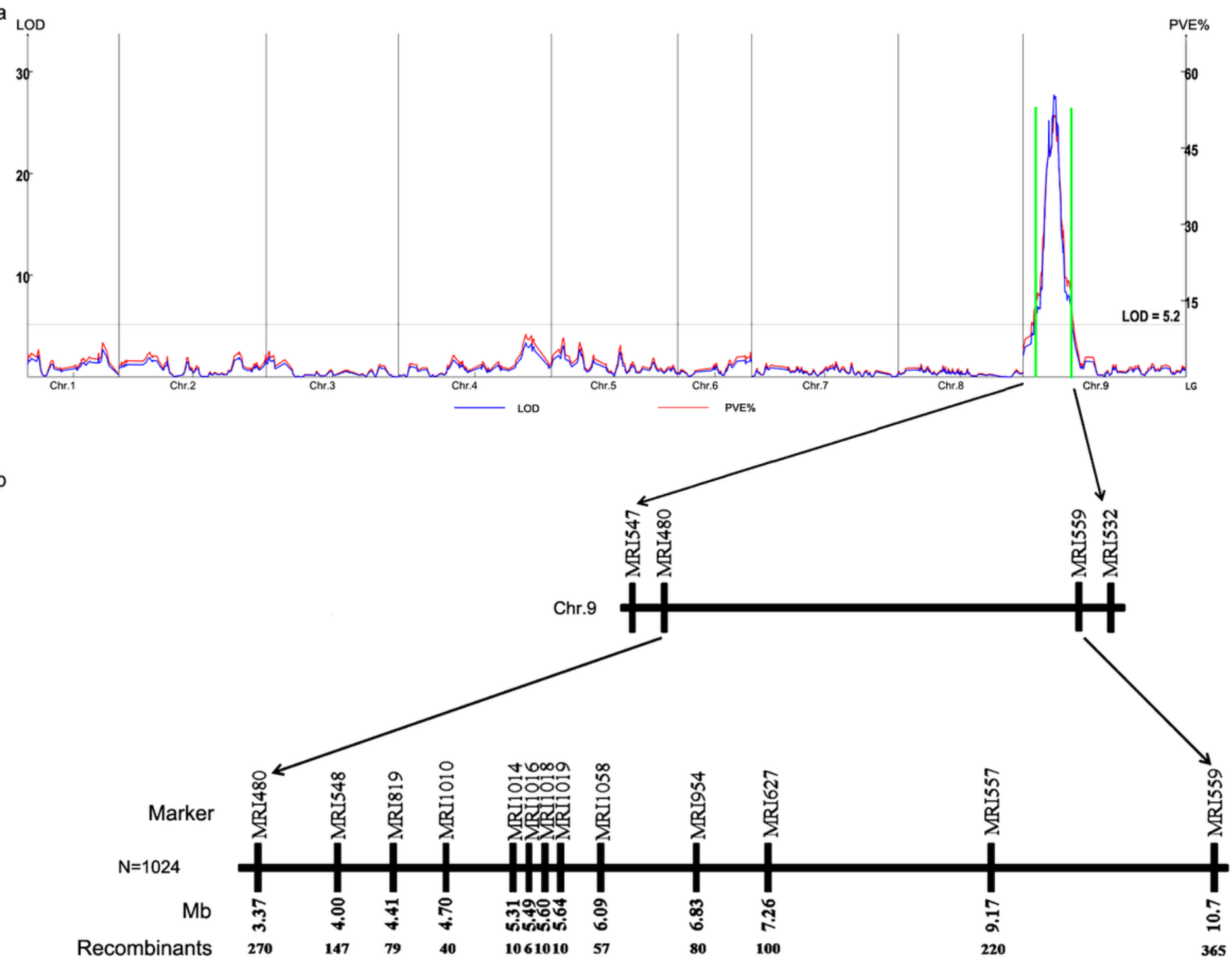

Figure 3

Fine mapping of qPH9. a qPH9 was preliminarily mapped on Chr. VIII with a physical interval of $9.2 \mathrm{Mb}$. Scale of $\mathrm{Y}$-axis on the left showed LOD value, and the right showed PVE, scale of X-axis showed the chromosomes; The gray line represented the threshold value of QTL mapping, the blue line represented LOD value, and the red line represented PVE. b qPH9 was narrowed down to an interval of $281 \mathrm{~kb}$ between MRI1014 and MRI1018.The marker name, physical position and number of recombinants were indicated

\section{Supplementary Files}

This is a list of supplementary files associated with this preprint. Click to download.

- Table1.xlsx

- Table2.xlsx 
- SupplementaryTableS1.xlsx

- SupplementaryTableS2.xlsx

- SupplementaryTableS3.xlsx

- SupplementaryTableS4.xlsx

- SupplementaryTableS5.xlsx

- SupplementaryTableS6.xlsx

- SupplementaryTableS7.xlsx

- SupplementaryTableS8.xlsx

- SupplementaryTableS9.xIsx

- Fig.S1.tif

- Fig.S2.tif 